Research Paper

\title{
Reorganized Collagen in the Tumor Microenvironment of Gastric Cancer and Its Association with Prognosis
}

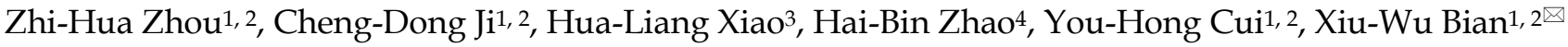 \\ 1. Institute of Pathology and Southwest Cancer Center, Southwest Hospital, Third Military Medical University, Chongqing, China; \\ 2. Key Laboratory of Tumor Immunopathology, Ministry of Education of China, Chongqing, China; \\ 3. Department of Pathology, Daping Hospital, Third Military Medical University, Chongqing, China; \\ 4. Department of Pathology, The 101 Hospital of PLA, Wuxi, Jiangsu Province, China. \\ $\square$ Corresponding author: Xiu-Wu Bian, Southwest Hospital, Third Military Medical University, Gaotanyan 30, Chongqing 400038, China. Phone/Fax: \\ 86-23-6875-4431; E-mail: bianxiuwu@tmmu.edu.cn \\ (1) Ivyspring International Publisher. This is an open access article distributed under the terms of the Creative Commons Attribution (CC BY-NC) license \\ (https:// creativecommons.org/licenses/by-nc/4.0/). See http://ivyspring.com/terms for full terms and conditions.
}

Received: 2016.11.23; Accepted: 2017.02.27; Published: 2017.06.01

\begin{abstract}
Collagen components in the tumor microenvironment substantially influence cancer pathogenesis and progression. Nevertheless, in gastric cancer, collagen status and its prognostic role remain unclear. Using picrosirius red staining and immunohistochemistry, we found that collagen deposition was significantly increased in gastric cancer when compared with non-neoplastic tissues, and in cancer stroma, more immature collagen components were present, suggesting a qualitative change. Furthermore, the morphology of collagen fibers could be weakly, moderately or strongly changed in gastric cancer; when weakly or moderately changed, they appeared similar to normal collagen fibers, except for a higher linearization and density; when strongly changed, they were thicker and less eosinophilic, sharply differently from their normal counterparts. In addition, we found abundant myofibroblasts and elevated expression of lysyl oxidase-like 2 (the enzyme that mediates crosslinking of collagen molecules) in cancer stroma, which might contribute to the increased collagen deposition and crosslinking. Last, five collagen architectural parameters (alignment, density, width, length and straightness) were analyzed with second harmonic generation imaging, a highly specific technology for detection of collagen fibers, and our data indicated that all the parameters were significantly increased in the tumor microenvironment. Of the five parameters, collagen width was the most powerful parameter in predicting 5-year overall survival, and increased collagen width was associated with reduced survival. The prognostic value of collagen width was superior to traditional clinicopathological parameters, and this was validated in two unrelated gastric cancer cohorts that contained 225 and 151 patients. Collectively, the collagen status (content, maturity, morphology and architecture) was profoundly reorganized in the tumor microenvironment of gastric cancer, and collagen width could serve as a valuable prognostic indicator.
\end{abstract}

Key words: gastric cancer; collagen fiber; immunohistochemistry; second harmonic generation; prognosis.

\section{Introduction}

The tumor microenvironment, comprising various cell components and non-cellular extracellular matrix (ECM), has profound impacts on tumor growth and spreading [1,2]. Fibrillar collagen is the dominant component of the ECM [3], and it has been increasingly recognized as an important contributor to cancer initiation and progression [4, 5]. For instance, densely deposited type I collagen in mouse mammary tissue can increase cancer formation by 3 times, and in this microenvironment, cancer cells exhibit a more aggressive behavior, leading to a 3-fold increase in lung metastasis [6]. Thus, a dense desmoplastic microenvironment with abundant collagen components promotes tumor progression, and collagen deposition is usually linked to adverse survival of patients $[7,8]$.

Globally, gastric cancer is the third leading cause of cancer-related death. Although the diagnosis and treatment of gastric cancer have greatly improved in recent decades, its prognosis remains very poor, with 
a 5-year survival rate of only $29.6 \%$ [9]. Histologically, there are varying amounts of stroma in gastric cancer, and the stroma substantially influences tumor development and progression. When evaluating the role of tumor stroma (microenvironment) in gastric cancer, most studies have primarily focused on stromal cells, while ECM elements, including fibrillar collagens, were seldom or insufficiently investigated. Previously, scirrhous gastric cancers (SGCs), a special gastric cancer type characterized by extensive stromal fibrosis and collagen deposition [10], are usually cited as an example to interpret the promotional role of collagen deposition in progression of gastric cancer, because the prognosis of SGC patients is very poor $[11,12]$. Nevertheless, SGCs only account for a low proportion $(10 \%)$ of all gastric cancer patients [10], and the status of collagen components in gastric cancer and how it is associated with patient outcomes remains to be elucidated.

In the present study, we systemically evaluated collagen status in gastric cancer, and our data revealed that collagen fibers were quantitatively and qualitatively reorganized. Moreover, we identified the structural features of collagen fibers, and our study indicated that collagen width could serve as a valuable prognostic indicator that is superior to traditional clinicopathological parameters.

\section{Methods}

\section{Patients}

Two gastric cancer cohorts were included in the present study. Cohort 1 contained 225 patients who underwent curative gastrectomy in The 101 Hospital of the People's Liberation Army from 2000 through 2011, and the median follow-up time was 54 months (range, 3-120 months). Cohort 2 was a validation cohort that included 151 patients who underwent curative gastrectomy in Southwest Hospital of Third Military Medical University from 2004 to 2007, and the median follow-up time was 52 months (range, 6-120 months). This study was approved by the Institutional Review Board/Ethics Committee from these two hospitals. Histological examination confirmed adenocarcinoma and R0 resection for all specimens. Patients who received preoperative chemotherapy were excluded. Medical records including pathology reports were reviewed to record patient sex, age, tumor location, tumor size, differentiation, Lauren classification, invasion depth of tumor, lymph node metastasis, and clinical stage. The American Joint Commission on Cancer TNM staging system was applied to determine the clinical stage of the tumor [13]. Follow-up data were collected by registered telephone, mail, or outpatient service.
The overall survival (OS) was defined as the interval between the date of the operation and gastric cancer-related death, while the disease-free survival (DFS) was calculated from the operation to the time of recurrence.

\section{Tissue microarray construction}

Specimens from 225 gastric cancer patients (cohort 1) and 22 cases of non-neoplastic mucosa were used to construct microarrays. Tissue cores $1 \mathrm{~mm}$ in diameter were punched from tumor blocks, and for each patient, two distinct tissue cores were sampled. Prior to punch, each region for sampling was reviewed to confirm its representativeness. Finally, the tissue microarray was constructed using a Veridiam Semi-Automated Tissue Arrayer (VTA-100).

\section{Immunohistochemistry (IHC) and assessment}

Tissue microarrays were serially cut into 4- $\mu$ m-thick sections for IHC of type I collagen, C-terminal propeptide of procollagen 1A1 (PICP), N-terminal propeptide of procollagen 1A1 (PINP), lysyl oxidase (LOX), lysyl oxidase-like 2 (LOXL2), and a-smooth muscle actin (a-SMA); details for these antibodies are shown in Table 1. Briefly, sections were deparaffinized and hydrated, followed by antigen retrieval $\left(95{ }^{\circ} \mathrm{C}, 15\right.$ minutes). After quenching endogenous peroxidase activity, sections were incubated with primary antibodies for 12 hours at 4 ${ }^{\circ} \mathrm{C}$. After being rinsed with wash buffer, sections were incubated with an anti-mouse/rabbit polymer kit (Envision Plus; Dako) for 30 minutes at room temperature. Thereafter, slides were visualized with chromogen (3,3-diaminobenzidine) and counterstained with hematoxylin. Negative controls were prepared by omitting primary antibodies, and the other procedures were identical to the test group.

Table 1. Antibodies used (source, clone and dilution)

\begin{tabular}{|c|c|c|c|c|c|}
\hline Antibody & Company & $\begin{array}{l}\text { Clone and } \\
\text { dilution }\end{array}$ & Species & $\begin{array}{l}\text { Catalog } \\
\text { number }\end{array}$ & Site \\
\hline a-SMA & $\begin{array}{l}\text { Maixin, } \\
\text { Fuzhou, } \\
\text { China }\end{array}$ & $\begin{array}{l}1 \mathrm{~A} 4 \\
1: 100\end{array}$ & Mouse & Kit-0006 & cytoplasmic \\
\hline LOX & $\begin{array}{l}\text { Santa Cruz, } \\
\text { Dallas, USA }\end{array}$ & $\begin{array}{l}\text { F-8 } \\
1: 100\end{array}$ & Mouse & sc-373995 & cytoplasmic \\
\hline LOXL2 & $\begin{array}{l}\text { Santa Cruz, } \\
\text { Dallas, USA }\end{array}$ & $\begin{array}{l}\text { Polyclone } \\
1: 200\end{array}$ & Goat & sc- -48723 & cytoplasmic \\
\hline $\begin{array}{l}\text { Type I } \\
\text { collagen }\end{array}$ & $\begin{array}{l}\text { Abcam, } \\
\text { Cambridge, UK }\end{array}$ & $\begin{array}{l}\text { EPR7785 } \\
1: 1500\end{array}$ & Rabbit & Ab138492 & extracellular \\
\hline PICP & $\begin{array}{l}\text { Santa Cruz, } \\
\text { Dallas, USA }\end{array}$ & $\begin{array}{l}\text { Polyclone } \\
1: 200\end{array}$ & Goat & sc- 25973 & extracellular \\
\hline PINP & $\begin{array}{l}\text { Santa Cruz, } \\
\text { Dallas, USA }\end{array}$ & $\begin{array}{l}\text { Polyclone } \\
1: 200\end{array}$ & Goat & sc- 8782 & extracellular \\
\hline
\end{tabular}

Abbreviations: a-SMA, a-smooth muscle actin; PICP, C-terminal propeptide of procollagen 1A1; PINP, N-terminal propeptide of procollagen 1A1 
IHC in tissue microarrays was evaluated according to staining area, and the positive percentage was determined as a percentage of positive area relative to the entire tumor area using Image J (version 1.48v, NIH, Bethesda USA). The case was regarded as positive when the positive area $\geq$ $10 \%$. This evaluation system was applied for assessing the staining of PICP, PINP, LOX and LOXL2, and a-SMA staining was evaluated by counting the positive percentage $(0-100 \%)$ of stromal fibroblasts.

\section{Picrosirius red staining and quantification}

Sections from the tissue microarray were stained with $0.1 \%$ picrosirius red (Direct Red 80; Sigma Aldrich). Stained sections were observed and imaged with an Olympus microscope (BX 51) equipped with a cross-polarizer. Images were thresholded and then analyzed for pixel density to quantify collagen content using Image J (version 1.48v, NIH, Bethesda USA).

\section{Second harmonic generation imaging of collagen fibers with a multiphoton microscope}

Second harmonic generation (SHG) imaging is a newly developed technology to examine noncentrosymmetric structures, which interact with multiphoton laser light, emitting SHG signals that can be detected [14]. In tissues, fibrillar collagens can be specifically detected by SHG imaging without a staining requirement, and the resulting images are high-resolution and quantifiable [15-17]. In the present study, SHG imaging was performed to detect collagen fibers in gastric cancer. SHG images were acquired with an LSM780 NLO microscope (Carl Zeiss AG, Germany) equipped with a Plan Apochromat 10× (NA 0.45) objective and a mode-locked Ti:Sapphire laser (690-1040 nm, <140fs; $90 \mathrm{MHz}$; Coherent Chameleon). Unfixed, hydrated tissue microarrays were observed with polarized laser light at a wavelength of $830 \mathrm{~nm}$, and the SHG signal was detected with a filter $(415 \mathrm{~nm})$. Images of $2048 \times 2048$ pixels (pixel size: $0.59 \mu \mathrm{m}$ ) were captured using Zen lite 2011 software (Carl Zeiss).

Prior to quantification of the collagen fibers, all SHG images were transformed into 8-bit images and thresholded between 10 and 255 gray levels to eliminate background noise. For each tissue core, five representative regions were used for analysis, and each region was $512 \times 512$ pixels. The quantitative parameters included overall density and overall alignment of collagen fibers, as well as parameters for single collagen fibers, including length, width and straightness. These collagen parameters are typically employed to characterize collagen organization in various cancer types $[16,17]$. The overall collagen density was determined in the entire tumor area of each tissue core with ImageJ (version $1.48 \mathrm{v}, \mathrm{NIH}$, Bethesda USA) as described above. The other collagen parameters (alignment, length, straightness, and width) were assayed using CT-FIRE, an open-source software package specifically designed for automatic quantification of collagen fibers within SHG images (http://loci.wisc.edu/software/ctfire) [18]. Of these parameters, alignment reflects the overall orientation of all collagen fibers, and its value ranges from 0 to 1 , where 1 indicates that all fibers are aligned at the same angle. Straightness also ranges from 0 to 1 , and to calculate this parameter, the linear length of the fiber was divided by the distance along the fiber.

\section{Statistics}

Correlations were examined using Pearson $\chi^{2}$ and Fisher exact tests. Comparison between two groups was carried out with an unpaired $t$-test. To evaluate the predictive value of collagen parameters for 5-year OS, receiver operating characteristic (ROC) curve analysis was employed to calculate the sensitivity and specificity, and the Youden index was estimated to determine the optimal cutoff value. Overall survival was determined using the Kaplan-Meier method, and survival curves were compared using the log-rank test. A Cox hazard regression analysis was carried out to determine the independent prognostic factors. Statistical analysis was performed using SPSS software (version 17.0) and Graphpad prism 5.0.

\section{Results}

\section{Increased stromal collagen deposition in gastric cancer}

Collagen content is an important aspect of collagen status. To characterize collagen status in gastric cancer, we first detected the collagen content in cancer stroma (from cohort 1) and in non-neoplastic gastric mucosa. Picrosirius red staining showed that there were few collagen fibers in non-neoplastic gastric mucosa, and these fibers were thin and sparsely distributed. However, in gastric cancer there was a marked increase of collagen deposition, and the collagen fibers were densely arranged, exhibiting a thick appearance (Figure 1a, b). As type I collagen is the most abundant collagen type in stroma, we further detected type I collagen with IHC. Consistent with the picrosirius red staining, IHC revealed a greater amount of type I collagen in cancer stroma than in the non-neoplastic tissues (Figure 1a, c). Therefore, this significant increase of collagen deposition in cancer stroma demonstrated a profound change in collagen relative to the normal counterpart. 
a
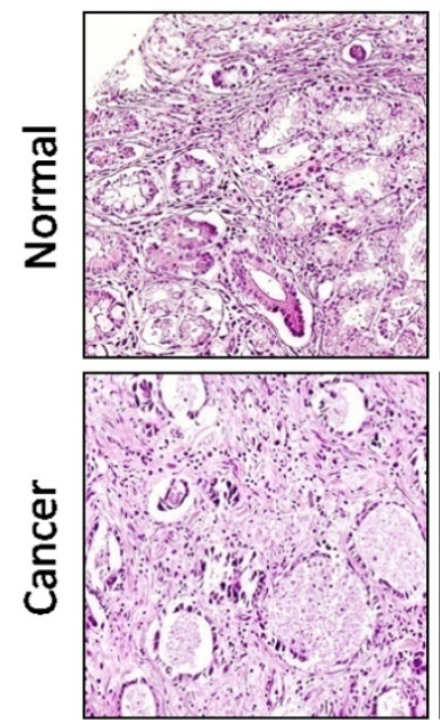

$H \& E$
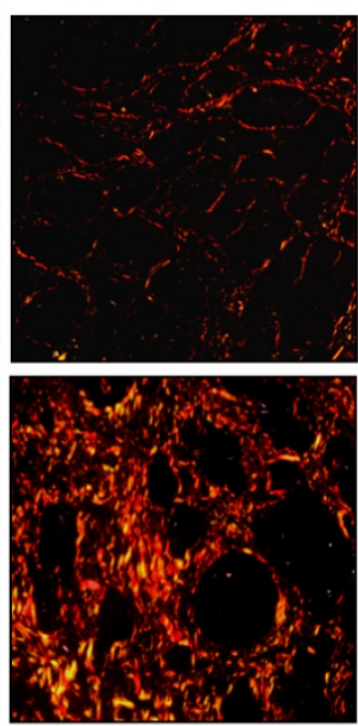

Picrosirius-red
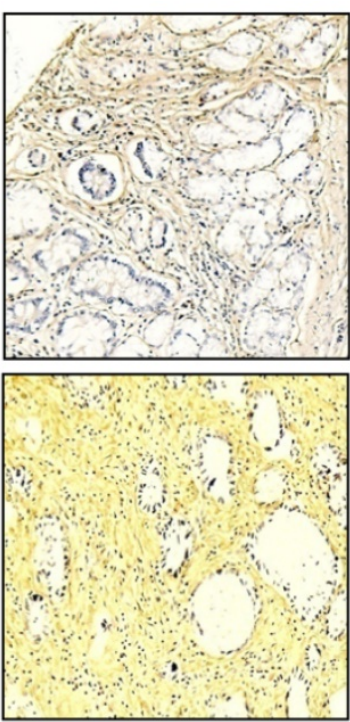

Collagen I b

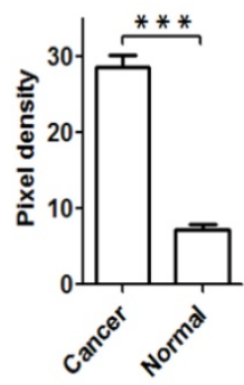

C

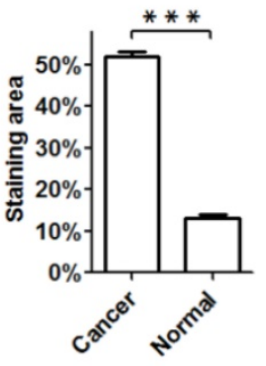

Figure 1. Increased stromal collagen deposition in gastric cancer. a. Normal mucosa and gastric cancer were stained with picrosirius red to visualize collagen, and type I collagen was labeled using immunohistochemistry. b. Picrosirius red quantification showed that the collagen content was significantly increased in stroma of gastric cancer when compared with normal mucosa. $\mathbf{c}$. The area of type I collagen stained by immunohistochemistry was also increased in gastric cancer. $* * * P<0.001$.

a

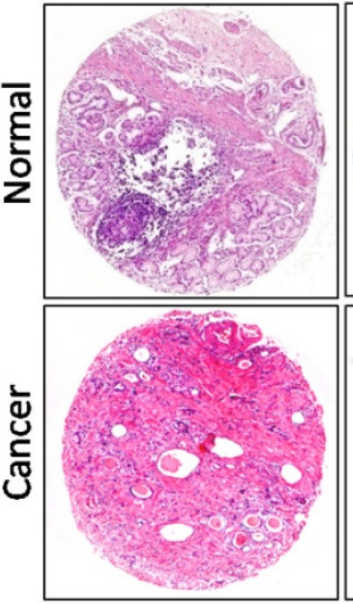

$H \& E$

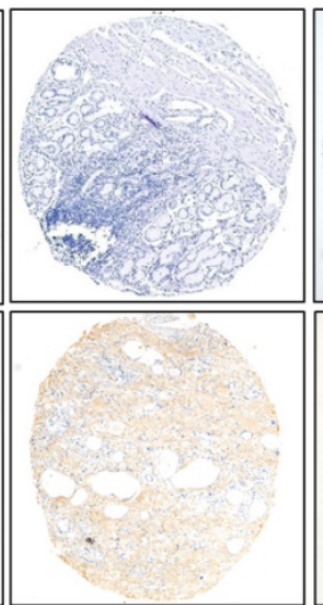

PINP

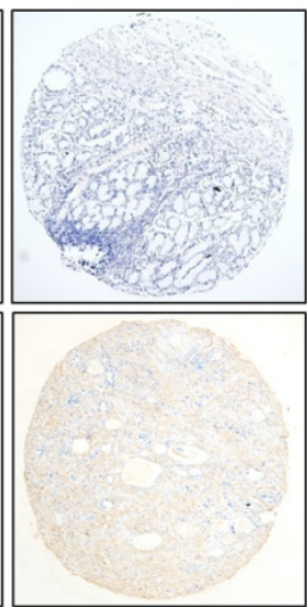

PICP b

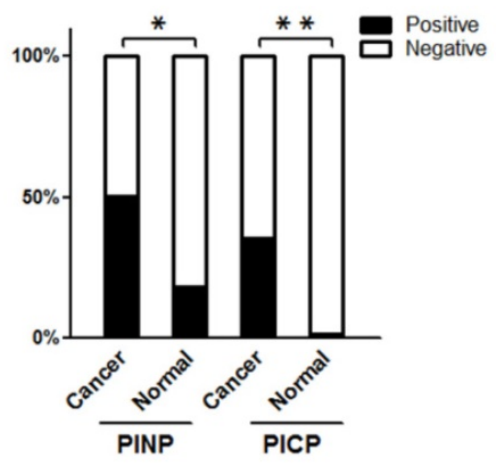

Figure 2. The presence of immature collagen in the stroma of gastric cancer. a.Immunohistochemistry of immature precursors of type I collagen (PINP and PICP). Both PINP and PICP were observed in cancer stroma, and in the normal mucosa, neither of these precursors were expressed. b. Stromal expression of PINP and PICP was significantly elevated in cancer tissue when compared with normal mucosa. $* P<0.05, * * P<0.01$. Abbreviations: PICP, C-terminal propeptide of type I procollagen; PINP, N-terminal propeptide of type I procollagen.

\section{Immaturity of collagen components in gastric cancer}

The mature forms of type I collagen molecules are derived from its precursor, type I procollagen, which contains a large amino-terminal propeptide and a carboxy-terminal propeptide (named PINP and PICP, respectively). When procollagen is secreted into the extracellular space, PINP and PICP are cleaved by proteolytic enzymes to form mature collagen molecules $[19,20]$. Using IHC, we labeled PINP and $\mathrm{PICP}$ in gastric cancer (cohort 1 ) and in non-neoplastic mucosa. Consequently, we found that both PINP and
PICP were present in the extracellular space (Figure 2a), and in cancer tissues, PINP and PICP were more highly expressed than in non-neoplastic mucosa (PINP: $50.6 \%$ vs $18.2 \%, P=0.035$; PICP: $35.5 \%$ vs $4.5 \%$, $P=0.003$; Figure $2 b$ ). Therefore, an increased number of immature collagen molecules were present in the tumor microenvironment, suggesting a qualitative change in stromal collagen in gastric cancer.

\section{Morphological alterations of collagen fibers in gastric cancer}

Collagen reorganization probably leads to 
morphological changes. Thus, we observed the morphological features of collagen fibers in detail. In normal gastric mucosa, collagen fibers were fine and wavy, with strong eosinophilia (Figure 3a). However, in gastric cancer (cohort 1), collagen fibers exhibited various morphological changes, and the 225 cases in cohort 1 could be divided into three subgroups according to the morphological alterations of collagen fibers. The first subgroup included 56 cases, which contained sparse, thin and eosinophilic collagen bundles in the stroma. Except for a more linearized appearance, the collagen fibers exhibited morphology similar to that of normal mucosa. Thus, the morphological alterations in this group were weak (Figure $3 b$ ). The second subgroup contained 113 cases, and in these cases, collagen fibers were also eosinophilic, but they exhibited a higher density and linearization than their normal counterparts (Figure 3c). Accordingly, morphological alterations in the second group were moderate. The third subgroup also contained 56 cases, and in these cases, there was also a marked increase in collagen density. Moreover, the morphology of collagen fibers was strikingly changed; these fibers were thick, highly linearized and irregularly aligned, and they exhibited less eosinophilia than their normal counterparts, showing a pink or pale staining (Figure $3 \mathrm{~d}$ ). Therefore, the morphological alterations were strong in the third subgroup. The morphological observations suggested that collagen reorganization varied among the different cases of gastric cancer.

\section{Increased myofibroblasts and collagen cross-linking enzymes in gastric cancer}

Collagen components in stroma are primarily produced by activated fibroblasts (myofibroblasts)
[21], which specifically express a-SMA [22]. We used IHC to detect a-SMA-positive myofibroblasts in the stroma of gastric cancer and non-neoplastic mucosa. As a result, we found more myofibroblasts in gastric cancer than in the non-neoplastic mucosa (Figure 4a, b). Thus, an increased number of collagen-producing myofibroblasts may be a crucial cause of increased collagen deposition in gastric cancer.

During the assembly of collagen fibers, LOX family molecules link collagen molecules and regulate their stability $[23,24]$. It has been reported that LOX and LOXL2 play important roles in collagen crosslinking and profoundly contribute to collagen reorganization in tumor stroma [25-28]. Considering this, we detected the expression of LOX and LOXL2 in gastric cancer using IHC. We found that LOX was mainly expressed in epithelial cells, and stromal expression was weak or absent in cancer and non-neoplastic tissues (Figure 4a). Neither epithelial nor stromal expression of LOX was significantly different between the cancer and non-neoplastic tissues (epithelial expression: $P=0.242$; stromal expression: $P=0.858$; Figure $4 \mathrm{c}$ ). In contrast, we found that LOXL2 was primarily expressed in stromal cells, and epithelial expression of LOXL2 was weak in both groups. In gastric cancer, stromal expression of LOXL2 was significantly stronger than in non-neoplastic mucosa $(P=0.001$; Figure $4 a, 4 d)$, but epithelial expression was comparable between the two groups $(P=0.566$; Figure $4 \mathrm{a}, 4 \mathrm{~d})$. Therefore, in the tumor microenvironment, there was excessive expression of LOXL2, which might enhance the cross-linking of collagen molecules, leading to dense arrangement of collagen fibers.

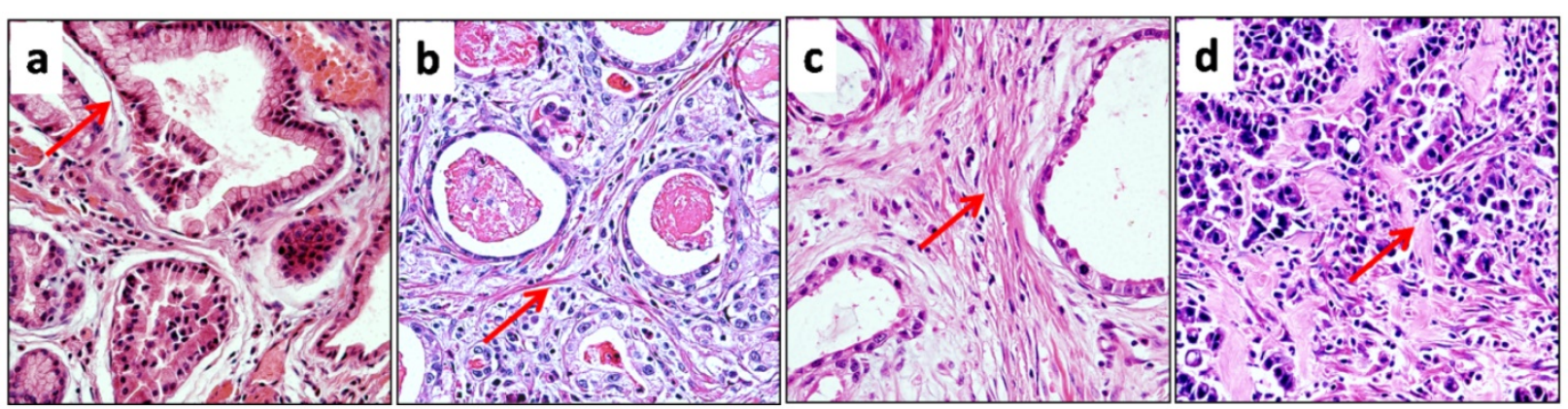

Figure 3. The morphology of collagen fibers changed differently in different cases of gastric cancer. a. In normal mucosa, sparse collagen fibers were present around glands, and the fibers were fine, wavy and eosinophilic (red arrow). b. In cancer stroma, collagen fibers displayed a weak change in morphology. These fibers were also thin and eosinophilic, but they were more linearized than their normal counterparts (red arrow). c. Collagen fibers in cancer stroma exhibited a moderate morphologic change, and they remained eosinophilic (red arrow). However, the density of collagen was significantly increased, and collagen fibers were straighter. d. The morphology of collagen fibers was strongly changed in cancer stroma, and there was also a marked increase in collagen density. Collagen fibers were very thick or sheet-like, and they were less eosinophilic than their normal counterparts, exhibiting a pink or pale appearance (red arrow). 
a
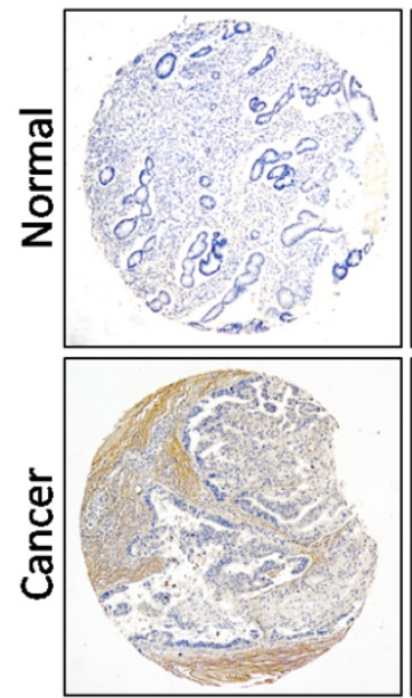

$\alpha-S M A$
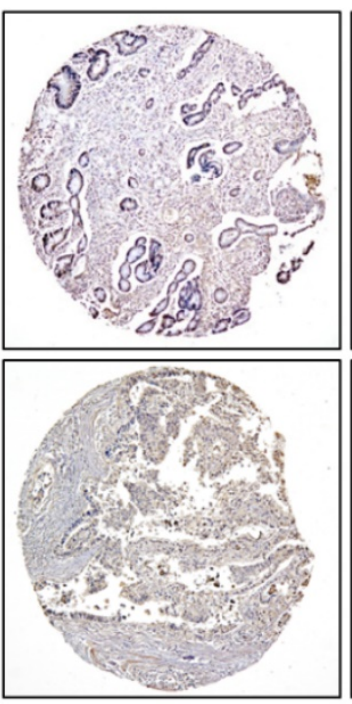

LOX

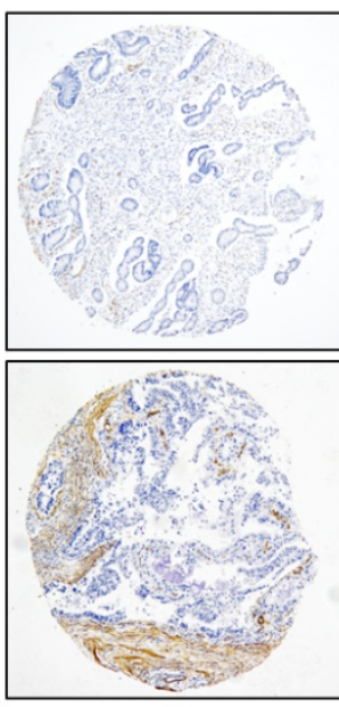

b

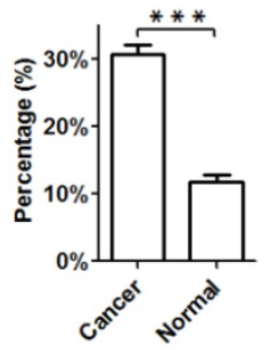

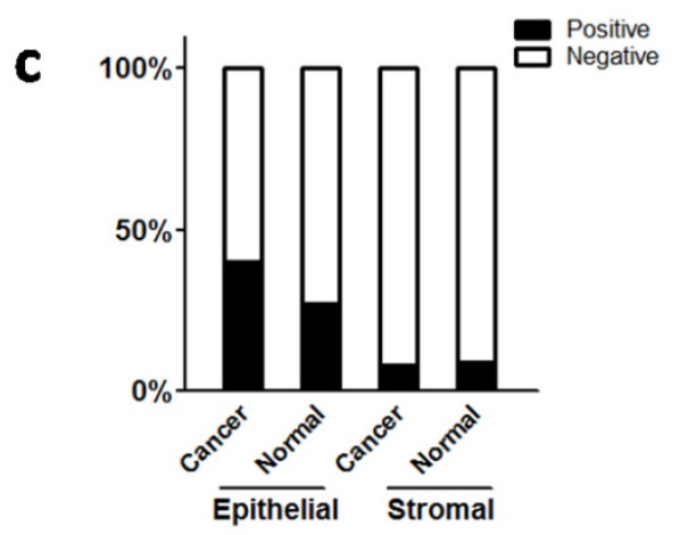

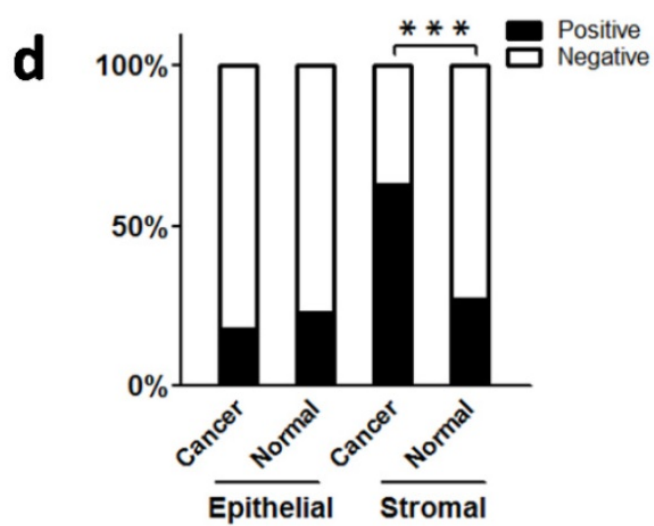

Figure 4. Increased myofibroblasts and expression of collagen crosslinking enzymes (LOX and LOXL2) in cancer stroma. a. Immunohistochemical staining to label a-SMA, LOX and LOXL2 in gastric cancer and normal mucosa. b. There were more $\alpha$-SMA-positive fibroblasts (myofibroblasts) in cancer stroma than in normal mucosa. c. For LOX, neither epithelial expression nor stromal expression differed between the cancerous and normal tissues. d. For LOXL2, there was no significant difference in epithelial expression between the cancerous and normal tissues, whereas stromal expression of LOXL2 in cancer tissues was higher. Abbreviations: $\alpha$-SMA, $\alpha$-smooth muscle actin; LOX, lysyl oxidase; LOXL2, lysyl oxidase-like 2. $* * * P<0.001$.

Quantitative analysis of stromal collagen fibers in gastric cancer

To quantitatively characterize changes in collagen fibers in gastric cancer, we determined quantitative parameters of the collagen fibers using SHG imaging, which is of high specificity and resolution for the detection of collagen fibers. These collagen parameters included alignment, density, length, width, and straightness, parameters which are frequently applied for characterization of collagen fibers [16, 17]. When the open-source software CT-FIRE is used, the collagen fibers in SHG images can be automatically extracted and analyzed for various parameters (Figure 5a). As expected, compared with their normal counterparts, collagen fibers in gastric cancer showed a significant increase in alignment, length, width and straightness (Figure $5 b)$. The collagen density was quantified using Image
$\mathrm{J}$, and it was also significantly elevated in cancer stroma (Figure 5b). Accordingly, these data suggested that the structure and organization of collagen fibers in the tumor microenvironment of gastric cancer had been markedly changed.

\section{The prognostic value of collagen parameters in gastric cancer}

We then used ROC curves to screen collagen parameters that were useful in prognosis evaluation, and 5-year OS was employed as an end point. Consequently, we found that three collagen parameters (density, length and width) were able to predict patient survival, and collagen alignment and straightness were not good predictors (Figure 6a, Table 2). Furthermore, we identified collagen width as the most powerful factor for prognosis prediction, and the area under the curve was 0.741 (Table 2). 
Then, we divided all the 225 cases (cohort 1 ) into a wide collagen group (93 cases) and a thin collagen group (132 cases) according to the collagen width (cutoff value: 4.02). Our data indicated that the wide collagen group contained more diffuse type gastric cancer $(P<0.001)$, and the cases in this group showed a poorer differentiation $(P<0.001)$, deeper mural invasion $(P<0.001)$, increased lymph node metastasis $(P<0.001)$, worse clinical stage $(P<0.001)$ and a higher rate of recurrence $(P<0.001)$ when compared with the thin collagen group (Table 3). Furthermore, the wide collagen group had more adverse prognoses than the thin collagen group (Figure 6b; 5-year OS: $20.4 \%$ vs $65.2 \%, \mathrm{P}<0.001$; 5 -year DFS: $20.4 \%$ vs $54.5 \%$, $P<0.001$ ). Employing a multivariate analysis, we found that classification based on collagen width was an independent prognostic factor for gastric cancer (Table 4), and its prognostic value was more powerful than traditional clinicopathological variables, such as tumor invasion depth or node metastasis (Table 4).
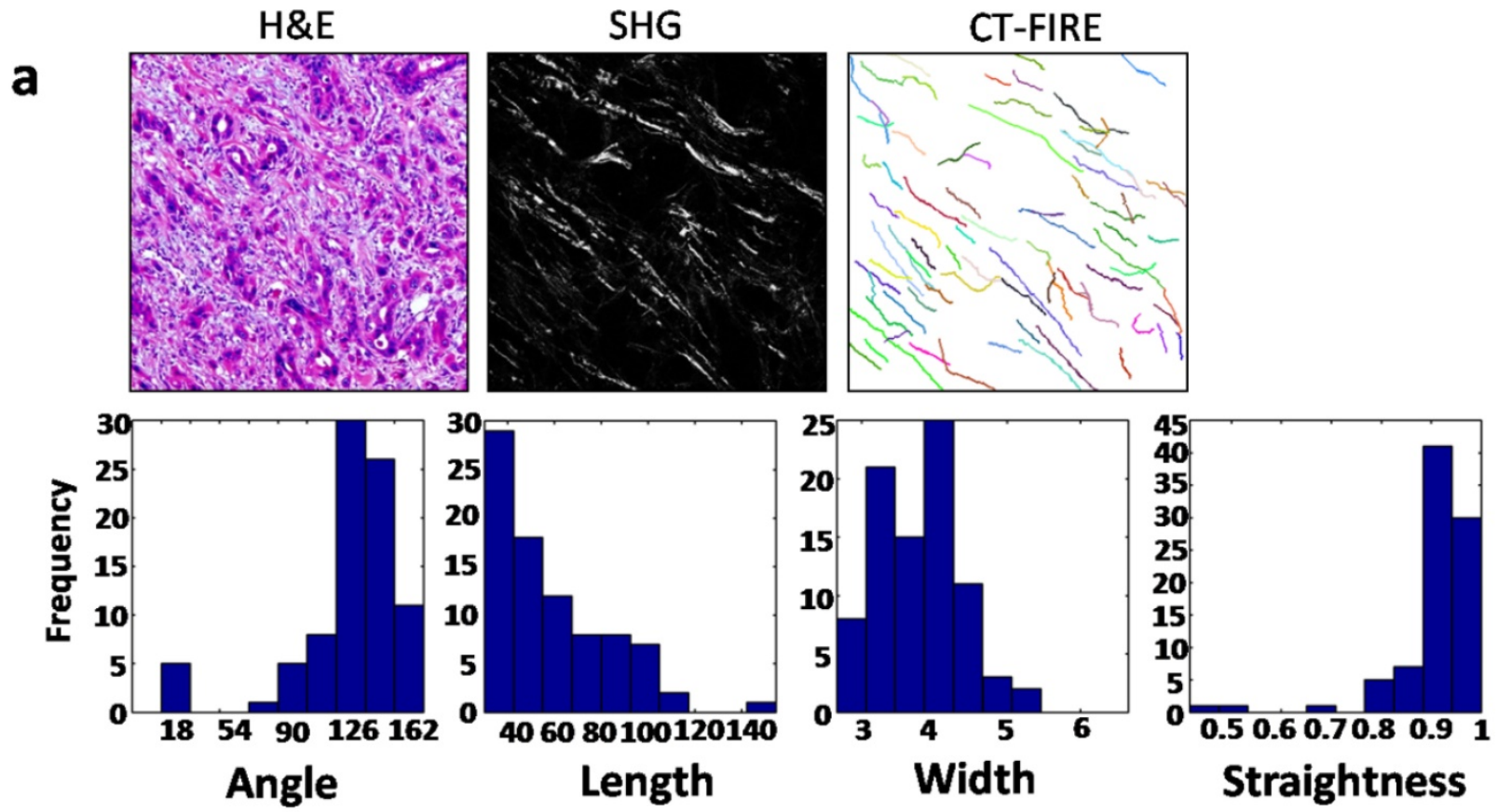

b
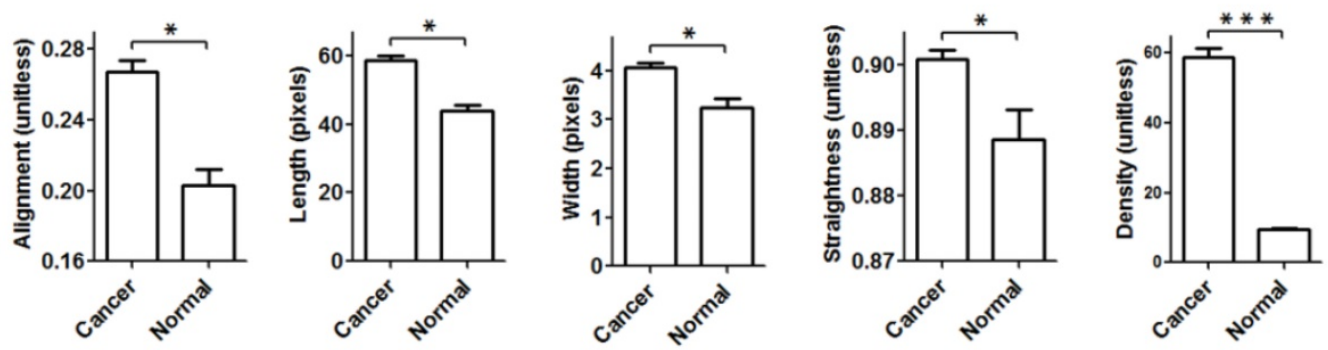

Figure 5. Second harmonic generation (SHG) imaging and quantitative analysis of collagen fibers. a. Collagen fibers were specifically visualized with an SHG multiphoton microscope, and collagen fibers were automatically extracted for analysis using the open-source software CT-FIRE. Then, histograms were generated to show the distribution of various parameters in each SHG image. b. Quantitative parameters including alignment, length, width, straightness and density were increased significantly in cancer stroma when compared with normal mucosa. Abbreviations: SHG, second harmonic generation. $* P<0.05$, $* * * P<0.001$.

Table 2. Prognostic value of collagen parameters in evaluating 5 -year overall survival.

\begin{tabular}{|c|c|c|c|c|c|c|}
\hline Collagen parameters & Area under the curve & $95 \%$ Confidence interval & $P$ value & Youden's index & Sensitivity & Specificity \\
\hline Width & 0.741 & $0.675-0.807$ & $<0.001$ & 0.436 & 0.617 & 0.819 \\
\hline Length & 0.672 & $0.602-0.742$ & $<0.001$ & 0.307 & 0.517 & 0.790 \\
\hline Density & 0.632 & $0.559-0.706$ & 0.001 & 0.350 & 0.617 & 0.733 \\
\hline Straightness & 0.525 & $0.450-0.601$ & 0.516 & 0.094 & 0.208 & 0.886 \\
\hline Alignment & 0.509 & $0.433-0.596$ & 0.810 & 0.079 & 0.717 & 0.362 \\
\hline
\end{tabular}


a

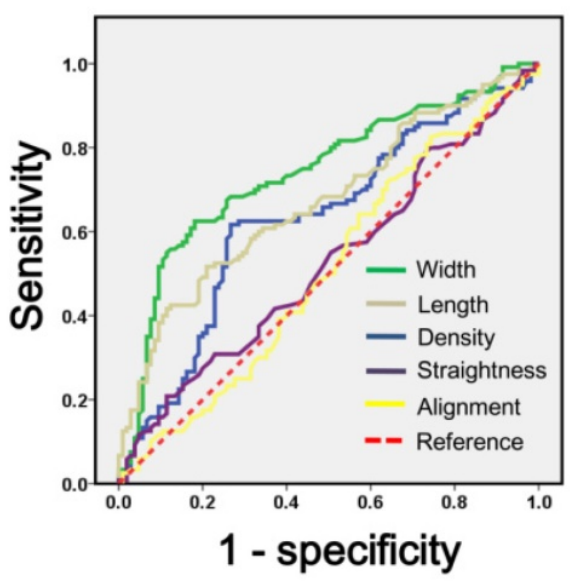

b

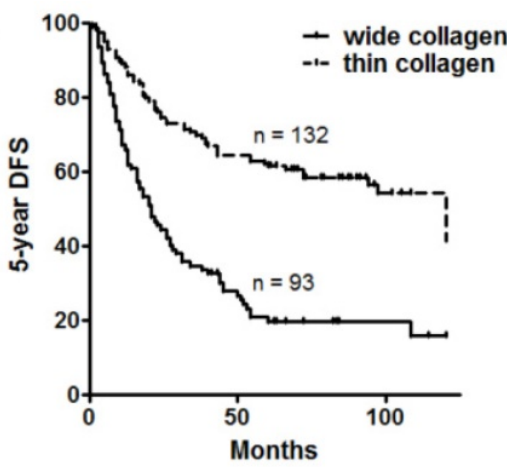

C

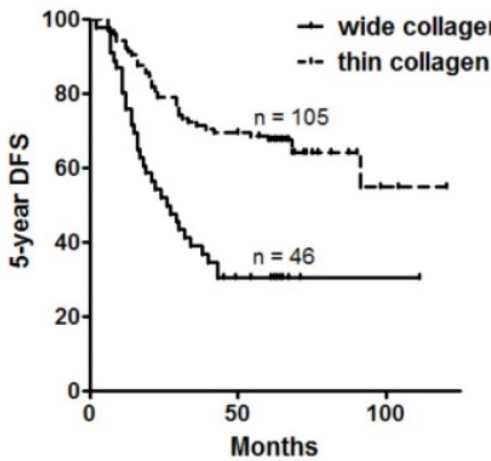

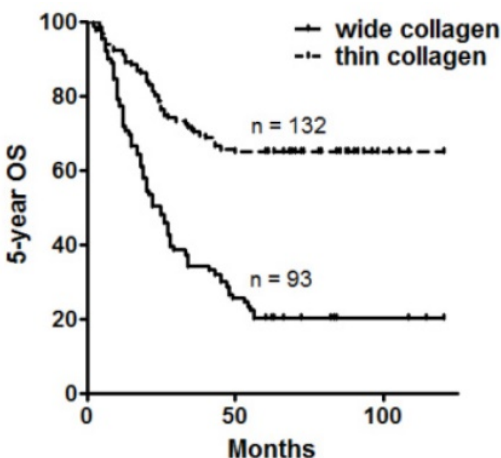

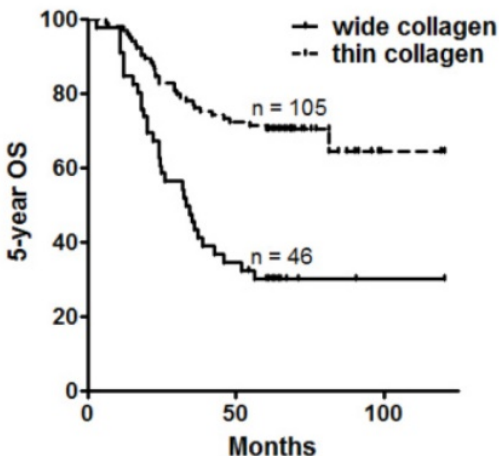

Figure 6. The prognostic value of collagen parameters in gastric cancer. a. ROC curves were applied to evaluate the value of five collagen parameters in predicting the 5 -year OS of gastric cancer patients, and collagen width was the most powerful factor. b. Cohort 1 was categorized into a wide collagen group and a thin collagen group according to collagen width, and the wide collagen group had a lower 5-year OS rate and 5-year DFS rate than the thin collagen group. c. The prognostic value of collagen width was confirmed in the validation cohort (cohort 2), which was unrelated to cohort 1 . The wide collagen group also exhibited a poorer outcome in this cohort. Abbreviations: OS, overall survival; DFS, disease-free survival.

Table 3. The association between collagen width and clinicopathological variables in cohort 1.

\begin{tabular}{|c|c|c|c|}
\hline Factors & $\begin{array}{l}\text { Wide collagen } \\
\text { group }\end{array}$ & $\begin{array}{l}\text { Thin collagen } \\
\text { group }\end{array}$ & $P$ value \\
\hline Gender & & & 0.862 \\
\hline Male & 70 & 98 & \\
\hline Female & 23 & 34 & \\
\hline Age & & & 0.166 \\
\hline$<60 y$ & 46 & 53 & \\
\hline$\geq 60 y$ & 47 & 79 & \\
\hline Location & & & 0.664 \\
\hline Upper & 37 & 60 & \\
\hline Middle & 15 & 15 & \\
\hline Lower & 33 & 48 & \\
\hline Diffuse & 8 & 9 & \\
\hline Tumor Size & & & 0.314 \\
\hline$\leq 3 \mathrm{~cm}$ & 32 & 58 & \\
\hline$\leq 6 \mathrm{~cm}$ & 43 & 55 & \\
\hline$>6 \mathrm{~cm}$ & 18 & 19 & \\
\hline $\begin{array}{l}\text { Tumor } \\
\text { differentiation }\end{array}$ & & & $<0.001$ \\
\hline Well & 1 & 24 & \\
\hline Moderate & 22 & 53 & \\
\hline Poor & 70 & 55 & \\
\hline Histological type & & & $<0.001$ \\
\hline Intestinal & 22 & 77 & \\
\hline Diffuse & 71 & 55 & \\
\hline pT stage & & & $<0.001$ \\
\hline $\mathrm{T} 1$ & 1 & 15 & \\
\hline $\mathrm{T} 2$ & 8 & 32 & \\
\hline T3 & 58 & 45 & \\
\hline $\mathrm{T} 4$ & 26 & 40 & \\
\hline pN stage & & & $<0.001$ \\
\hline
\end{tabular}

\begin{tabular}{llll}
\hline N0 & 15 & 62 & \\
N1 & 19 & 21 & \\
N2 & 21 & 30 & $<0.001$ \\
N3 & 38 & 19 & \\
Clinical stage & & & \\
I & 3 & 34 & $<0.001$ \\
II & 25 & 46 & \\
III & 65 & 52 & \\
Recurrence & & & \\
Presence & 74 & 60 & \\
Absence & 19 & 72 &
\end{tabular}

Finally, we validated the prognostic value of collagen width in an unrelated gastric cancer cohort (cohort 2), which contained 151 cases and was from a different medical center. We classified these patients according to collagen width, and the cutoff value was 4.02. Consistently, this classification was also significantly associated with traditional prognostic variables (Table 5), and a larger collagen width also predicted a lower 5-year OS $(30.4 \%$ vs $69.5 \%, P<$ 0.001 ) or 5 -year DFS (30.4\% vs $65.7 \%, P<0.001$; Figure $6 c)$. In addition, this categorization could also serve as an independent prognostic factor for gastric cancer (Table 5), and its prognostic value remained more effective than traditional clinicopathological variables (Table 6). 
Table 4. Independent prognostic factors for cohort 1 when collagen width was included for multivariable analysis.

\begin{tabular}{|c|c|c|c|c|c|c|}
\hline \multirow[t]{2}{*}{ Factors } & \multicolumn{3}{|c|}{ Overall survival } & \multicolumn{3}{|c|}{ Disease-free survival } \\
\hline & HR & $95 \% \mathrm{CI}$ & $P$ value & HR & $95 \% \mathrm{CI}$ & $P$ value \\
\hline Collagen width & 2.673 & $1.759-4.062$ & $<0.001$ & 2.297 & $1.546-3.414$ & $<0.001$ \\
\hline T stage & 1.571 & $1.122-2.200$ & 0.009 & 1.385 & $1.010-1.898$ & 0.043 \\
\hline $\mathrm{N}$ stage & 1.447 & $1.068-1.960$ & 0.017 & 1.439 & $1.079-1.920$ & 0.013 \\
\hline Age & 0.485 & $0.322-0.731$ & 0.001 & 0.535 & $0.366-0.783$ & 0.001 \\
\hline
\end{tabular}

Table 5. The association between collagen width and clinicopathological variables in cohort 2.

\begin{tabular}{|c|c|c|c|}
\hline Factors & $\begin{array}{l}\text { Wide collagen } \\
\text { group }\end{array}$ & $\begin{array}{l}\text { Thin collagen } \\
\text { group }\end{array}$ & $P$ value \\
\hline Gender & & & 0.615 \\
\hline Male & 33 & 71 & \\
\hline Female & 13 & 34 & \\
\hline Age & & & 0.533 \\
\hline$<60 \mathrm{y}$ & 22 & 56 & \\
\hline$\geq 60 \mathrm{y}$ & 24 & 49 & \\
\hline Location & & & 0.633 \\
\hline Upper & 12 & 23 & \\
\hline Middle & 22 & 48 & \\
\hline Lower & 12 & 31 & \\
\hline Diffuse & 0 & 3 & \\
\hline Tumor Size & & & 0.007 \\
\hline$\leq 3 \mathrm{~cm}$ & 13 & 52 & \\
\hline$\leq 6 \mathrm{~cm}$ & 24 & 47 & \\
\hline$>6 \mathrm{~cm}$ & 9 & 6 & \\
\hline $\begin{array}{l}\text { Tumor } \\
\text { differentiation }\end{array}$ & & & 0.001 \\
\hline Well & 0 & 10 & \\
\hline Moderate & 12 & 50 & \\
\hline Poor & 34 & 45 & \\
\hline Histological type & & & $<0.001$ \\
\hline Intestinal & 12 & 62 & \\
\hline Diffuse & 34 & 43 & \\
\hline pT stage & & & 0.014 \\
\hline $\mathrm{T} 1$ & 1 & 17 & \\
\hline $\mathrm{T} 2$ & 9 & 32 & \\
\hline T3 & 21 & 37 & \\
\hline $\mathrm{T} 4$ & 15 & 19 & \\
\hline pN stage & & & 0.523 \\
\hline No & 21 & 51 & \\
\hline N1 & 7 & 18 & \\
\hline N2 & 7 & 21 & \\
\hline N3 & 11 & 15 & \\
\hline Clinical stage & & & 0.072 \\
\hline I & 6 & 32 & \\
\hline II & 19 & 37 & \\
\hline III & 21 & 36 & \\
\hline Recurrence & & & $<0.001$ \\
\hline Presence & 32 & 36 & \\
\hline Absence & 14 & 69 & \\
\hline
\end{tabular}

\section{Discussion}

To our knowledge, we are one of the few, if not the first to systematically elucidate collagen status in gastric cancer. Histopathological evaluation of gastric cancer primarily focuses on the epithelial elements, although tumor stroma and ECM have been increasingly recognized as important factors influencing carcinogenesis and progression. Collagen fibers are the most abundant components of ECM [3]; however, collagen status in gastric cancer remains unclear. Here, our study combined histopathological methods and SHG imaging to determine stromal collagen status (content, maturity, morphology and architecture) in gastric cancer, and our data revealed that collagen components were substantially altered (reorganized) in the tumor microenvironment.

In the present study, we found that collagen components were quantitatively and qualitatively changed in gastric cancer. Using picrosirius red staining and IHC, we first determined collagen content in gastric cancer, and a marked increase in collagen deposition was observed (quantitative change). Previous studies found that densely deposited collagen could increase the formation of breast cancer, and this collagen-rich microenvironment could promote invasion and metastasis [6]. Similarly, increased collagen deposition might facilitate the development and progression of gastric cancer. We also identified qualitative changes in gastric cancer; PINP and PICP were found in cancer stroma, indicating the presence of immature collagen molecules. During the assembly of mature collagen fibers, PINP or PICP is cleaved and degraded from procollagen molecules, and the presence of PINP or PICP indicates that procollagen is not sufficiently modified in a timely manner, suggesting a vigorous synthesis of type I collagen [29, 30]. If PINP or PICP remain attached to procollagen molecules, the shape and solubility of collagen molecules will be influenced [19, 20]. Thus, the immature collagen components may exhibit different physical and chemical features when compared with mature collagen, and the impact of immature collagen on cancer cells remains unclear.

To date, the morphology and structure of collagen fibers in gastric cancer have not been identified. Increased collagen deposition, enhanced collagen cross-linking and the presence of immature collagen molecules likely lead to morphologic changes in collagen fibers. Indeed, our study revealed that the morphology of collagen fibers could be weakly, moderately or strongly changed. When weakly or moderately changed, the morphology of 
collagen fibers in cancer stroma were similar to those in non-neoplastic mucosa, but the linearization or (and) density of collagen fibers were increased; when strongly changed, the content, thickness and eosinophilia were significantly altered. Thus, collagen fibers in gastric cancer were morphologically distinct from their normal counterparts, and they were differently altered among different cases of gastric cancer, reflecting the heterogeneity of collagen reorganization. Furthermore, we quantitatively determined structural signatures of collagen fibers using SHG imaging, which is a high-resolution imaging method specific for collagen detection. Our data indicated that all parameters of collagen fibers (alignment, density, length, width and straightness) were significantly elevated in cancer stroma, suggesting a marked structural change. Therefore, our study revealed the morphological and structural changes in collagen fibers in gastric cancer, and these changes might serve as ancillary histological signs for distinguishing benign and malignant lesions of the stomach.

Currently, accumulating evidence indicates that collagen signatures have a great impact on tumor progression. In vitro studies found that increased diameter or length of collagen fibers could increase the invasive distance of breast cancer cells, and linearized collagen fibers enhance the stiffness of the ECM, which in turn promotes cancer invasion [31]. Moreover, a recent study indicated that elongated collagen fibers in tumor stroma of head and neck, esophageal and colorectal cancersis linked to a poor outcome [32]. In the present study, we found that three structural parameters were associated with prognosis in gastric cancer; increased density, length or width of collagen fibers could predict a poor outcome. Among these parameters, collagen width was the most powerful prognostic factor, and its prognostic value was superior to traditional clinicopathological parameters. This was validated in two independent gastric cancer cohorts (from west and east China). Therefore, through quantification of collagen features, our study provided a useful prognostic indicator for gastric cancer. As SHG imaging can be directly used to image hematoxylin and eosin stained sections, structural analysis for collagen fibers might be combined with other clinicopathological variables to evaluate patient outcomes.

Why is collagen reorganized in gastric cancer? We believe that the increase in activated fibroblasts (myofibroblast) was a crucial contributor to collagen reorganization. These myofibroblasts express a-SMA, and they are the major source of various ECM components, including fibrillar collagens [21]. Consistently, we found significantly increased numbers of a-SMA-positive myofibroblasts in the stroma of gastric cancer. Furthermore, our study demonstrated that stromal expression of LOXL2, an important enzyme for collagen cross-linking, was more evident in gastric cancer. This may enhance collagen cross-linking, leading to a higher density of collagen, and it likely increases the length and width of collagen fibers. Previous studies have found that targeting the tumor microenvironment with certain enzymatic agents or small molecules could remodel tumor stroma and decrease intestinal fluid pressure, promoting the delivery of chemotherapeutic agents $[33,34]$. Recently, it was reported that LOXL2 antibodies could disrupt orientation and width of collagen fibers, ultimately resulting in decreased tumor growth [35]. Therefore, targeting activated myofibroblasts and collagen cross-linking enzymes may be a promising treatment for gastric cancer.

In conclusion, our study revealed that collagen components are quantitatively and qualitatively reorganized in the tumor microenvironment of gastric cancer. We established an association between collagen features and prognosis, and collagen width was identified as a useful prognostic indicator for gastric cancer.

Table 6. Independent prognostic factors for cohort 2 when collagen width was included for multivariable analysis.

\begin{tabular}{|c|c|c|c|c|c|c|}
\hline \multirow[t]{2}{*}{ Factors } & \multicolumn{3}{|c|}{ Overall survival } & \multicolumn{3}{|c|}{ Disease-free survival } \\
\hline & HR & $95 \% \mathrm{CI}$ & $P$ value & HR & $95 \% \mathrm{CI}$ & $P$ value \\
\hline Collagen width & 2.093 & $1.191-3.678$ & 0.010 & 2.201 & $1.254-3.862$ & 0.006 \\
\hline T stage & 2.040 & $1.409-2.952$ & $<0.001$ & 1.964 & $1.380-2.795$ & $<0.001$ \\
\hline Gender & 0.580 & $0.332-1.011$ & 0.055 & 0.573 & $0.339-0.969$ & 0.038 \\
\hline Tumor size & 1.623 & $1.079-2.442$ & 0.020 & 1.715 & $1.143-2.574$ & 0.009 \\
\hline
\end{tabular}

\section{Acknowledgments}

This work was supported by the National Basic Research Program of China (973 Program, No. 2010CB529400).

\section{Competing Interests}

The authors have declared that no competing interest exists. 


\section{References}

1. Joyce JA, Pollard JW. Microenvironmental regulation of metastasis. Nat Rev Cancer. 2009; 9: 239-52

2. Bissell MJ, Hines WC. Why don't we get more cancer? A proposed role of the microenvironment in restraining cancer progression. Nat Med. 2011; 17: 320-9.

3. Egeblad M, Rasch M, Weaver VM. Dynamic interplay between the collagen scaffold and tumor evolution. Curr Opin Cell Biolo.2010; 22: 697-706.

4. Bonnans C, Chou C, Werb Z. Remodelling the extracellular matrix in development and disease. NatRevMolCell Biology. 2014; 15:786-801.

5. Levental KR, Yu H, Kass L, et al. Matrix crosslinking forces tumor progression by enhancing integrin signaling. Cell. 2009; 139: 891-906.

6. Provenzano PP, Inman DR, Eliceiri KW, et al. Collagen density promotes mammary tumor initiation and progression. BMC Med. 2008; 6:11.

7. Conklin MW, Keely PJ. Why the stroma matters in breast cancer: insights into breast cancer patient outcomes through the examination of stromal biomarkers . Cell Adh Migr. 2012; 6:249-60.

8. Whatcott CJ, Diep CH, Jiang P, et al. Desmoplasia in primary tumors and metastatic lesions of pancreatic cancer. ClinCancer Res. 2015; 21: 3561-8.

9. [Internet] IARC: Lyon, France. GLOBOCAN 2012: estimated cancer incidence, mortality and prevalence worldwide in 2012, Section of cancer information. v1.2. http://globocan.iarc.fr.Default.aspx. Revised January 2015.

10. Duarte I, Llanos O. Patterns of metastases in intestinal and diffuse types of carcinoma of the stomach. Hum Pathol. 1981; 12: 237-42.

11. Maehara Y, Moriguchi S, Orita H, et al. Lower survival rate for patients with carcinoma of the stomach of Borrmann type IV after gastric resection. Surg Gynecol Obstet. 1992; 175:13-6.

12. Schauer M, Peiper M, Theisen J, et al. Prognostic factors in patients with diffuse type gastric cancer (linitis plastica) after operative treatment. Eur J Med Res. 2011; 16: 29-33.

13. Edge SB, Byrd DR, Compton CC, et al. AJCC cancer staging manual, 7th edn. New York, USA: Springer; 2009.

14. Campagnola PJ, Loew LM. Second-harmonic imaging microscopy for visualizing biomolecular arrays in cells, tissues and organisms. Nat Biotechnol. 2003; 21:1356-60

15. Conklin MW, Eickhoff JC, Riching KM, et al. Aligned collagen is a prognostic signature for survival in human breast carcinoma. Am J Pathol. 2011; 178:1221-32.

16. Drifka CR, Tod J, Loeffler AG, Liu Y, et al. Periductal stromal collagen topology of pancreatic ductal adenocarcinoma differs from that of normal and chronic pancreatitis. Modern Pathol. 2015; 28:1470-80.

17. Keikhosravi A, Bredfeldt JS, Sagar AK, et al. Second harmonic generation imaging of cancer. Methods Cell Biol. 2014; 123:531-46.

18. Bredfeldt JS, Liu Y, Pehlke CA, et al. Computational segmentation of collagen fibers from second-harmonic generation images of breast cancer. J Biomed Opt. 2014;19:16007.

19. Mouw JK, Ou G, Weaver VM. Extracellular matrix assembly: a multiscale deconstruction. Nat Rev Mol Cell Biol. 2014; 15:771-85.

20. Hulmes DJ. Building collagen molecules, fibrils, and suprafibrillar structures. J Struct Biol. 2002;137: 2-10.

21. Alexander J, Cukierman E. Stromal dynamic reciprocity in cancer: intricacies of fibroblastic-ECM interactions. CurrOpinCell Biol. 2016; 42:80-93.

22. Navab R, Strumpf D, Bandarchi B, et al. Prognostic gene-expression signature of carcinoma-associated fibroblasts in non-small cell lung cancer. Proc Natl Acad Sci U S A. 2011; 108:7160-5.

23. Csiszar K. Lysyl oxidases: a novel multifunctional amine oxidase family. Prog Nucleic Acid Res Mol Biol. 2001;70:1-32.

24. Cenizo V, André V, Reymermier C, et al. LOXL as a target to increase the elastin content in adult skin: a dill extract induces the LOXL gene expression. Exp Dermatol. 2006;15: 574-81.

25. Barker HA, Cox TR, Erler JT. The rationale for targeting the LOX family in cancer. Nat Rev Cancer. 2012; 12:540-52.

26. Cox TR, Bird D, Baker A, et al. LOX-mediated collagen crosslinking is responsible for fibrosis-enhanced metastasis. Cancer Res. 2013; 73: 1721-32.

27. Wong CC, Tse AP, Huang YP, et al. Lysyl oxidaselike 2 is critical to tumor microenvironment and metastatic niche formation in hepatocellular carcinoma. Hepatology. 2014, 60:1645-58.

28. Torres S, Garciapalmero I, Herrera M, et al. LOXL2 is highly expressed in cancer-associated fibroblasts and associates to poor colon cancer survival. Clin Cancer Res. 2015; 21: 4892-902.

29. Kauppila S, Stenback F, Risteli J, et al. Aberrant type I and type III collagen gene expression in human breast cancer in vivo. J Pathol. 1998; 186:262-8.

30. Berruti A, Piovesan A, Torta M, et al. Biochemical evaluation of bone turnover in cancer patients with bone metastases: relationship with radiograph appearances and disease extension. Br J Cancer. 1996, 73: 1581-7.

31. Insua-rodriguez J, Oskarsson T. The Extracellular Matrix in Breast Cancer. Adv Drug Deliv Rev. 2016; 97:41-55.

32. Hanley C J, Noble F, Ward M J, et al. A subset of myofibroblastic cancer-associated fibroblasts regulate collagen fiber elongation, which is prognostic in multiple cancers. Oncotarget. 2015;7: 6159-74.

33. Shields MA, Dangi-Garimella S, Redig AJ, et al. Biochemical role of the collagen-rich tumour microenvironment in pancreatic cancer progression. Biochem J. 2012; 441:541-52.
34. Lu J, Zhou S, Siech M, et al. Pancreatic stellate cells promote hapto-migration of cancer cells through collagen I-mediated signalling pathway. Br J Cancer. 2014;110:409-20.

35. Grossman M, Benchetrit N, Zhuravlev A, et al. Tumor cell invasion can be blocked by modulators of collagen fibril alignment that control assembly of the extracellular matrix. Cancer Res. 2016; 76: 4249-58. 